\title{
Do higher-risk patients benefit from off-pump coronary artery bypass grafting? Evidence from an ecologic analysis of randomized trials
}

\author{
Oliver Kuss, $\mathrm{PhD},{ }^{\mathrm{a}}$ and Jochen Börgermann, $\mathrm{MD}^{\mathrm{b}}$
}

Objective: It is commonly believed that especially higher-risk patients benefit from off-pump coronary artery bypass grafting. However, analyses from several registries give different results. A common shortcoming of all those analyses is the fact that they concentrate on evidence from nonrandomized trials.

\begin{abstract}
Methods: In an ecologic analysis, we included all randomized trials comparing the on- and off-pump techniques until January 2011. By logistic regression, we investigated whether the effect of off-pump surgery on mortality, myocardial infarction, stroke, and atrial fibrillation is modified across the range of the 3 risk factors: age, proportion of women, and ejection fraction.
\end{abstract}

Results: Eighty-six studies with a total population of 9906 patients reported on at least 1 risk factor and 1 outcome. We found a superiority of the off-pump technique in patients with lower ejection fraction values for the outcomes mortality and atrial fibrillation. No effect modification was seen for the risk factors age and proportion of women.

Conclusions: Our ecologic analysis of nearly 10,000 patients from 86 randomized trials found a superiority of the off-pump technique in patients with lower ejection fraction values, especially for the most valid outcome of mortality. As every ecologic analysis is prone to ecologic bias, a definite answer on the benefit of the off-pump technique in higher-risk patients can only be given by meta-analyses using individual patient data. (J Thorac Cardiovasc Surg 2011;142:e117-22)

It is commonly believed that especially patients with a higher baseline risk benefit from off-pump coronary artery bypass (OPCAB) grafting. As early as 2005, the International Society for Minimally Invasive Cardiothoracic Surgery issued a consensus statement stating that OPCAB should be considered in patients with a EuroSCORE greater than 5, age greater than 75 years, diabetes, renal failure, left ventricular dysfunction, left main disease, and in patients undergoing reoperation. ${ }^{1}$ Later reviews corroborated these recommendations. ${ }^{2-4}$ However, in our review of 35 propensity score analyses, ${ }^{5}$ we found very similar odds ratios in standard groups (odds ratio, 0.68 [95\% confidence intervals: $0.60,0.77]$ ) and high-risk groups (odds ratio, $0.71[0.66,0.78])$ in a pooled analysis for 11 short-term outcomes. A common shortcoming of all those analyses is the fact that they mostly concentrate on evidence from non-

\footnotetext{
From the Institute of Medical Epidemiology, Biostatistics, and Informatics, ${ }^{\text {a Faculty }}$ of Medicine, University of Halle-Wittenberg, Halle (Saale), Germany; and the Heart and Diabetes Center North Rhine-Westphalia, ${ }^{\text {b }}$ Department of Thoracicand Cardiovascular Surgery, Ruhr-University Bochum, Bad Oeynhausen, Germany. Disclosures: Authors have nothing to disclose with regard to commercial support.

Received for publication Nov 16, 2010; revisions received March 17, 2011; accepted for publication April 26, 2011; available ahead of print June 13, 2011.

Address for reprints: Oliver Kuss, PhD, Institute of Medical Epidemiology, Biostatistics, and Informatics, Medical Faculty, University of Halle-Wittenberg, Magdeburger Strasse 8, 06097 Halle (Saale), Germany (E-mail: Oliver.Kuss@medizin. uni-halle.de)

$0022-5223 / \$ 36.00$

Copyright (c) 2011 by The American Association for Thoracic Surgery doi:10.1016/j.jtcvs.2011.04.032
}

randomized trials, where treatment assignment might be biased by patient characteristics. We report here on an ecologic analysis of the possible modification of the OP$\mathrm{CAB}$ treatment effect through risk factors. The study is exclusively based on data from randomized controlled trials (RCTs).

\section{MATERIALS AND METHODS}

The included RCTs were collected in 2 steps. First, we included all RCTs from the most recent general systematic review of Møller and associates. ${ }^{6}$ Second, inasmuch as the Møller review includes RCTs only up to June 2007, we additionally performed a MEDLINE search in January 2011 for the keywords "Coronary Artery Bypass, Off-Pump [MeSH]," "off-pump [All Text]," “opcab [All Text]," "beating heart," "op-cab [All Text]," "off pump" [All Text], or "octopus [All Text]" where we restricted on RCTs in humans. ${ }^{7}$ Finally, we searched our personal files for additional studies. One of us (O.K.) read all studies in full text and extracted mean or median age, proportion of women, and ejection fraction $(\mathrm{EF})$. In cases in which the EF was only given in categories with percentages, we assumed values evenly distributed in categories and calculated the median value from the reported figures. For all analyses, we equated medians to means to describe the average value of the respective distribution. The risk factors age, proportion of women, and EF were chosen because the Møller article ${ }^{6}$ suggests that the respective data are available in the majority of studies. In terms of outcomes, we concentrated on the 4 short-term results: mortality, myocardial infarction, stroke, and atrial fibrillation. The former 3 were chosen because of their clinical relevance and the last 1 because of its nonsparse occurrence, thus providing sufficient power for analysis. Finally, information on risk factors and outcomes was also compared with our own database of RCTs. ${ }^{8}$ 


\section{Abbreviations and Acronyms \\ $\mathrm{EF} \quad=$ ejection fraction \\ $\mathrm{OPCAB}=$ off-pump coronary artery bypass \\ $\mathrm{RCT} \quad=$ randomized controlled trial}

To visualize associations between outcomes and risk factors, we drew scatter plots of treatment effect (measured as relative risks) versus the average value of the risk factor from each study. To assess the modifying effect of a risk factor on the treatment effect, we calculated-similarly to Puskas and coworkers ${ }^{4}$ - logistic regression models. These included the respective outcome (mortality, myocardial infarction, stroke, or atrial fibrillation) as the dependent variable. The binary variable treatment (off-pump vs on-pump), the respective risk factor (average age, proportion of women, or EF) on a continuous scale, and the interaction of both served as independent variables. The latter interaction assesses our main hypothesis of interest, because it measures whether treatment effects vary across the range of values of the respective risk factor. Additionally, our logistic regression models included a random intercept for each study to account for the correlation of pa-
TABLE 1. Distribution of risk factors (average values) in the 86 RCTs under study

\begin{tabular}{lccr}
\hline \multicolumn{1}{c}{ Risk factor } & N & Median & (Min, Q1, Q3, Max) \\
\hline Age (y) & 85 & 63.1 & $48.3,61.3,65.0,75.9$ \\
Proportion female (\%) & 82 & 20.4 & $0.6,16.9,25.2,66.2$ \\
EF $(\%)$ & 66 & 61.9 & $33.5,56.0,67.2,75.0$ \\
\hline
\end{tabular}

$R C T$, Randomized controlled trial; $E F$, ejection fraction.

tients within studies. Moreover, to interpret treatment effects as relative risks and not as odds ratios, we used a log link function (instead of the standard logit link function). To facilitate interpretation, we estimated the treatment effect for a set of a priori values of the respective risk factor and included these data in the scatter plots.

For each combination of risk factor and outcome, we calculated an additional logistic model without reference to the respective risk factor. This latter model gives the standard estimate for the treatment effect (assumed to be constant over the whole range of risk factors) and can be used for comparisons.

Finally, to measure the influence of all 3 risk factors simultaneously, we fitted a single model for each outcome that included all 3 risk factors and their 3 interactions with the risk factors as covariates.
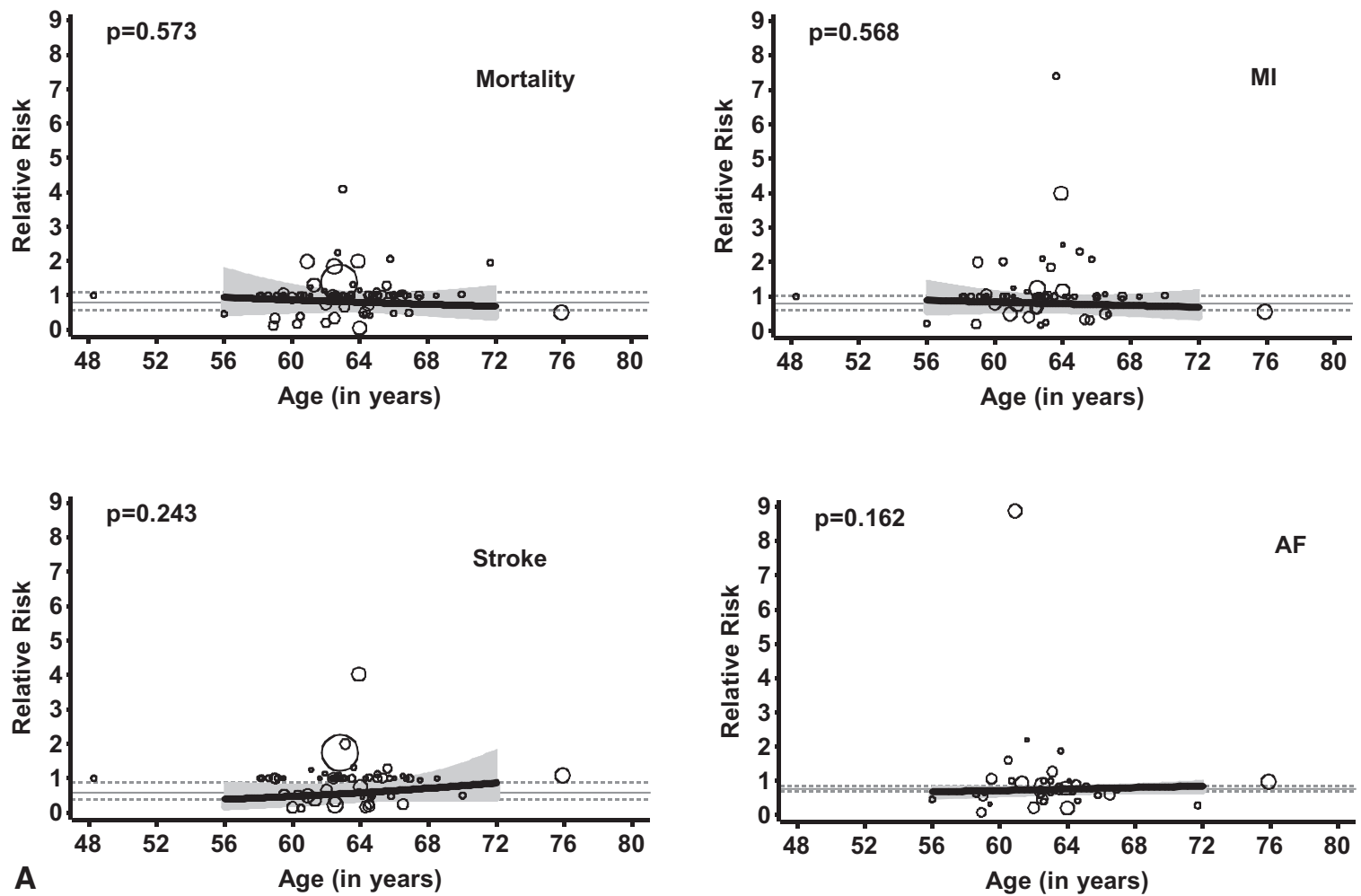

FIGURE 1. Scatter plots for each combination of risk factors (A, age; B, proportion female; C, ejection fraction) and outcome (mortality, myocardial infarction, stroke, and atrial fibrillation). Each plot gives 4 pieces of information. First, each circle represents a single study with its average value of the risk factor on the $\mathrm{x}$-axis and the relative risk for off-pump surgery on the $\mathrm{y}$-axis. Each circle area is proportional to the overall number of patients in the respective study. For plotting the circles (but not for statistical analysis), studies with no event in one or both treatment arms were corrected by the 0.5 -continuity correction. Second, the black line gives the relative risk of the respective outcome with off-pump treatment depending on its value for the respective risk factor. These lines are allowed to vary across the range of the risk factor, indicating a potential effect modification of the off-pump effect. Third, light gray lines depict the off-pump effect (with its $95 \%$ confidence interval) as calculated from a model that assumes a constant treatment effect across the range of the risk factor. Fourth, the given $P$ value stems from the test on the interaction of treatment and risk factor and can be interpreted as a test for no effect modification of the risk factor. $M I$, Myocardial infarction; $A F$, atrial fibrillation; $L V E F$, left ventricular ejection fraction. 

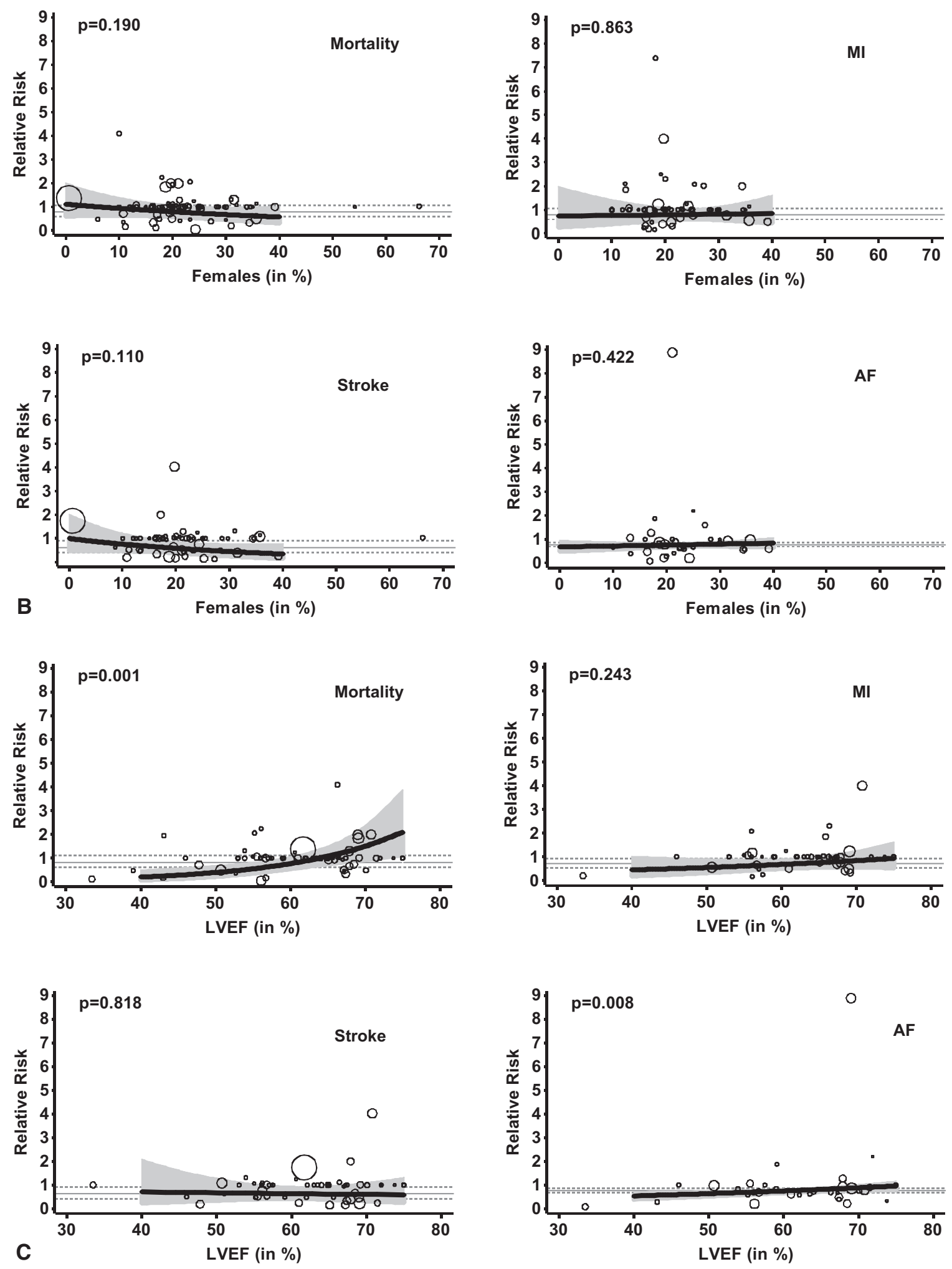

FIGURE 1. (continued).

It is essential to note that while all these models use outcome and treatment information on each individual patient (easily derived from the respective study's 4-fold table), the value of the respective risk factor (eg, age) is not available for individual patients. Instead, the average value from the respective study is used to describe the individual patient's age. By definition, this approach makes our study an ecologic 
TABLE 2. Estimates for the interaction of treatment and the respective risk factor for the 4 different outcomes in models for each risk factor separately and in a model in which all 3 risk factors enter simultaneously

\begin{tabular}{|c|c|c|c|c|c|}
\hline \multirow[b]{2}{*}{ Outcome } & \multirow[b]{2}{*}{ Risk factor } & \multicolumn{2}{|r|}{ Separate models } & \multicolumn{2}{|r|}{ Simultaneous model } \\
\hline & & $\mathbf{N}$ & $\begin{array}{l}\text { Interaction estimate } \\
(95 \% \mathrm{CI}), P \text { value }\end{array}$ & $\mathbf{N}$ & $\begin{array}{l}\text { Interaction estimate } \\
(95 \% \mathrm{CI}), P \text { value }\end{array}$ \\
\hline \multirow[t]{3}{*}{ Mortality } & Age (y) & 82 & $-0.020(-0.090,0.050), .573$ & 60 & $0.045(-0.046,0.135), .334$ \\
\hline & Proportion female $(\%)$ & 79 & $-0.017(-0.041,0.008), .190$ & 60 & $-0.017(-0.045,0.012), .256$ \\
\hline & $\mathrm{EF}(\%)$ & 64 & $0.066(0.027,0.106), .001$ & 60 & $0.074(0.028,0.120), .002$ \\
\hline \multirow[t]{3}{*}{ MI } & Age (y) & 61 & $-0.016(-0.070,0.039), .568$ & 44 & $0.005(-0.073,0.083), .901$ \\
\hline & Proportion female $(\%)$ & 57 & $0.003(-0.035,0.042), .863$ & 44 & $0.007(-0.046,0.061), .786$ \\
\hline & $\mathrm{EF}(\%)$ & 47 & $0.021(-0.014,0.056), 0.243$ & 44 & $0.024(-0.017,0.065), 0.249$ \\
\hline \multirow[t]{3}{*}{ Stroke } & Age (y) & 66 & $0.050(-0.034,0.134), .243$ & 50 & $0.107(-0.002,0.216), .053$ \\
\hline & Proportion female $(\%)$ & 63 & $-0.027(-0.059,0.006), .110$ & 50 & $-0.047(-0.087,-0.007), .021$ \\
\hline & $\mathrm{EF}(\%)$ & 53 & $-0.006(-0.054,0.043), .818$ & 50 & $0.004(-0.052,0.060), 0.884$ \\
\hline \multirow[t]{3}{*}{$\mathrm{AF}$} & Age (y) & 37 & $0.014(-0.006,0.033), .162$ & 31 & $0.036(0.006,0.066), .017$ \\
\hline & Proportion female $(\%)$ & 35 & $0.005(-0.008,0.018), .422$ & 31 & $0.003(-0.015,0.022), .711$ \\
\hline & $\mathrm{EF}(\%)$ & 32 & $0.016(0.004,0.028), .008$ & 31 & $0.029(0.014,0.044), .0001$ \\
\hline
\end{tabular}

$C I$, Confidence interval; $M I$, myocardial infarction; $A F$, atrial fibrillation.

one. To be concrete, we assume here that the effect of the risk factor on the outcome is the same, regardless of whether we observe the risk factor value for each individual patient or whether we observe only the average value from the study that the patient belongs to. We additionally refer to Appendix 1, where we illustrate this situation (and the corresponding danger of ecologic bias) by a simple example that mirrors the situation given here.

All estimates are given with their $95 \%$ confidence intervals; statistical analyses were conducted with SAS version 9.2. (SAS Institute, Inc, Cary, NC).

\section{RESULTS}

Eighty-six studies with a total population of 9906 patients reported on at least 1 risk factor and 1 outcome. Information on average age, proportion of women, and EF was available in 85,82 , and 66 studies, respectively. The distribution of the average values of risk factors is depicted in Table 1. In terms of outcomes, information on mortality, myocardial infarction, stroke, and atrial fibrillation was available in 83, 61, 65, and 37 studies, respectively. Figure 1, $A$ to $C$, shows the scatter plots for each combination of risk factor and outcome and Table 2 gives the respective parameter estimates.

As seen in the graphs (Figure 1, $A-C$ ), there is no effect modification by age or the proportion of women for any of the outcomes: Graphs are more or less parallel to the $\mathrm{x}$-axis, indicating a constant off-pump effect across the available range of the risk factor. As expected from previous results, there is solid evidence of an effect modification by EF: smaller EF values are associated with a larger superiority of the off-pump technique. This is especially true for the outcomes mortality and atrial fibrillation. However, there is also evidence that the off-pump technique is inferior with high EF values. In the models including all 3 risk factors simultaneously, estimates are qualitatively similar.

\section{COMMENT}

Our ecologic analysis of nearly 10,000 patients from 86 randomized trials found a superiority of the off-pump technique in patients with lower EF values, especially for the most valid outcome of mortality. However, no effect modification was seen for the risk factors age and proportion of women. These findings are partly in line with our observation of similar effects in higher-risk and standard populations from 35 propensity score analyses ${ }^{5}$ but contradicts the findings of others. Several reasons could explain the observed differences. The first concerns the populations under study. Registries and observational studies include the entire spectrum of patients treated during the observational period, that is, both the "healthiest" and the "sickest" patients. Randomized off-pump studies, however, continue to include healthier populations. This can be exemplified by analyzing the risk factors of patients in the RCTs: only 3 included trials had an average age of 70 years or more and the median age over all studies was 63 years. In contrast, in 2008 more than $40 \%$ of all cardiac surgical patients in Germany were older than 70 years. ${ }^{9}$ That is, although our study covers a wide range of values for the respective outcomes, it can provide only limited evidence for the groups of highest risk at the edge of the distributions. The second reason might be the different study designs. Whereas Puskas and associates ${ }^{4}$ included individual patient data, we had to resort to aggregated risk factor data, which makes our analysis vulnerable to ecologic bias.

\section{LIMITATION}

It is a limitation of our study reported here that we could use only aggregated risk factor data. It is widely known that such ecologic analyses are prone to the so-called "ecologic fallacy," which occurs when associations that exist on an aggregate level do not represent the true association on an 
individual level. ${ }^{10}$ This drawback in design, however, made it possible to include information from nearly all of the RCTs conducted worldwide up to now.

\section{CONCLUSIONS}

A definitive answer to the question under study can only be given by an individual data-based meta-analysis that collects individual data from as many RCTs as possible. We are aware of 1 attempt of such an analysis from a Dutch group that was reported as a poster abstract and as "work in progress." 11 The group collected 9 individual RCTs with more than 100 patients in 2004. Unfortunately, no further analyses from this data set were published. Those types of analyses and RCTs specifically designed to recruit high-risk groups such as the GOPCABE Study (German Off Pump Coronary Artery Bypass in Elderly Study; ClinicalTrials.gov number: NCT00719667) will finally answer the question we were asking in the title of this article.

\section{References}

1. Puskas J, Cheng D, Knight J, Angelini G, DeCannier D, Diegeler A, et al. Offpump versus conventional coronary artery bypass grafting: a meta-analysis and consensus statement from the 2004 ISMICS consensus conference. Innovations. 2005;1:3-27.

2. Rastan AJ, Walther T, Falk V, Lehmann S, Kempfert J, Mohr FW. Coronary artery bypass grafting on the beating heart in high-risk patients. Herz. 2007;32: 483-90.

3. Kerendi F, Morris CD, Puskas JD. Off-pump coronary bypass surgery for highrisk patients: only in expert centers? Curr Opin Cardiol. 2008;23:573-8.

4. Puskas JD, Thourani VH, Kilgo P, Cooper W, Vassiliades T, Vega JD, et al. Offpump coronary artery bypass disproportionately benefits high-risk patients. Ann Thorac Surg. 2009;88:1142-7.

5. Kuss O, von Salviati B, Börgermann J. Off-pump versus on-pump coronary artery bypass grafting: a systematic review and meta-analysis of propensity score analyses. J Thorac Cardiovasc Surg. 2010;140:829-35. 835.e1-13.

6. Møller CH, Penninga L, Wetterslev J, Steinbruchel DA, Gluud C. Clinical outcomes in randomized trials of off- vs. on-pump coronary artery bypass surgery: systematic review with meta-analyses and trial sequential analyses. Eur Heart J. 2008;29:2601-16.

7. Møller CH, Penninga L, Wetterslev J, Steinbrüchel DA, Gluud C. Off-pump versus on-pump coronary artery bypass grafting for ischaemic heart disease (Protocol). Cochrane Database Syst Rev. 2008;Issue 3. Art. No.: CD007224.

8. Kuss O, Legler T, Börgermann J. Do treatments effects differ between randomized trials and propensity score analyses in similar populations? Evidence from a meta-propensity score analysis in off-pump versus on-pump coronary artery bypass surgery. J Clin Epidemiol. 8 April 2011 [Epub ahead of print]

9. Gummert JF, Funkat A, Beckmann A, Schiller W, Hekmat K, Ernst M, et al. Cardiac surgery in Germany during 2008. A report on behalf of the German Society for Thoracic and Cardiovascular Surgery. Thorac Cardiovasc Surg. 2009;57: 315-23.

10. Tu JV, Ko DT. Ecological studies and cardiovascular outcomes research. Circulation. 2008;118:2588-93.

11. Kluytmans M, Van der Heijden GJMG, Borst C, Grobbee DE. Equal effects of off-pump and on-pump coronary artery bypass surgery (work in progress). Eur J Epidemiol. 2006;21(S1):99.

\section{APPENDIX 1. AN EXEMPLARY ILLUSTRATION OF ECOLOGIC BIAS}

For the sake of illustration, assume that 3 RCTs have been conducted to compare the off-pump with the onpump technique. The 3 trials differ with respect to the age of the included patients; the first trial included patients from 55 to 65 years, the second trial patients from 65 to 75 years, and the third trial patients from 75 to 85 years.

Appendix Figures 1 to 3 give hypothetical results of these 3 trials to describe a potential ecologic bias. These 3 figures are very similar to the figure in the main article. They display the values of a risk factor (here: age) on the $\mathrm{x}$-axis and the relative risk for an outcome on the $\mathrm{y}$-axis. The crucial difference is that we here have access to the original patient data, which are displayed by small plus signs (+) in the figures. In the original analysis in the main article, we have no information on individual data; we only know the average (or aggregated) value of the respective study populations, which are given as circles in Appendix Figures 1 to 3.

We additionally give regression lines that describe the association of age and the effect separately in the 3 trials (full lines) and in the ecologic (averaged) situation (broken line). Ecologic bias will be present if the results from the individual data differ from those of the averaged data, that is, if the study's regression lines differ from the averaged regression line.

There are several situations that can arise when we want to assess the effect of the risk factor age on the risk of the outcome.

Appendix Figure 1 gives a situation in which there will be ecologic bias. In all 3 RCTs we have an increasing risk with increasing age. However, as in our ecologic analysis, we only observe the average ages per study (that is, the 3 circles); we wrongly judge (by referring to the broken regression line) that there is no effect of age.

In Appendix Figure 2 there will be no ecologic bias. We find a clear risk increase with increasing age; however, this increase can also be seen when we only observe the average age values per study. That is, we will find the same effect of age in the individual and also in the aggregated data, resulting in the absence of ecologic bias.

In Appendix Figure 3 there will also be no ecologic bias. When we look at the individual observations, there is no effect of age in any of the 3 RCTs. Going to the average data, we also find no effect of age. 


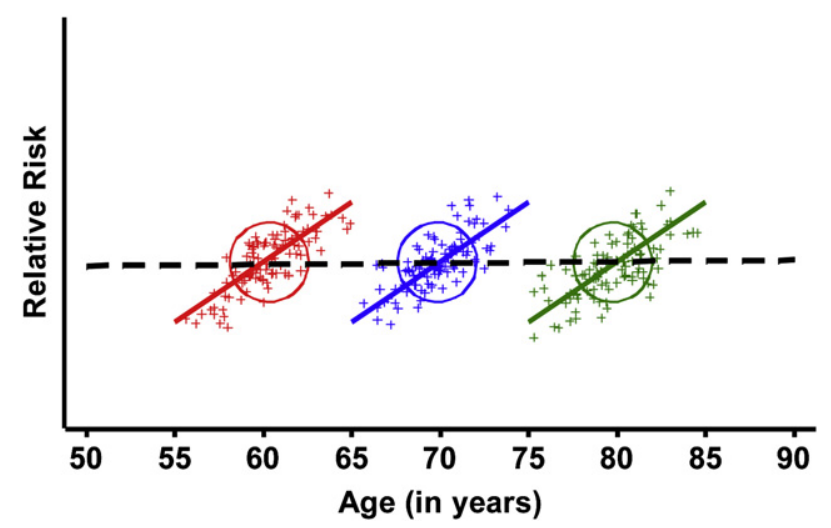

APPENDIX FIGURE 1. Ecologic bias.

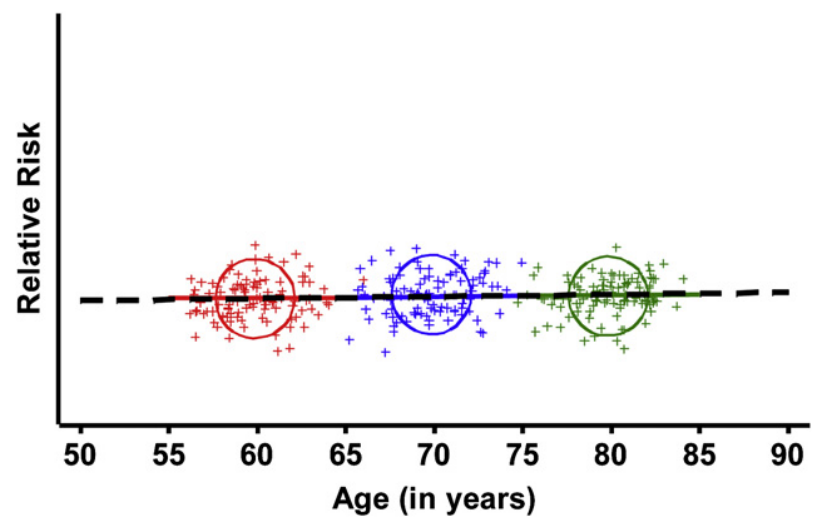

APPENDIX FIGURE 3. No ecologic bias.

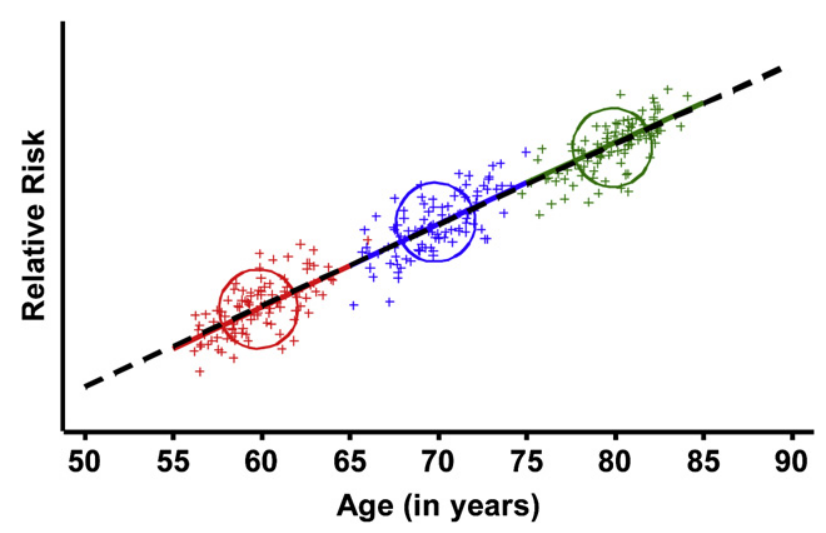

APPENDIX FIGURE 2. No ecologic bias.

e122 The Journal of Thoracic and Cardiovascular Surgery • September 2011 